\section{Molecular genetics of primary congenital glaucoma}

\footnotetext{
M. Sarfarazi

I. Stoilov

Molecular Ophthalmic

Genetics Laboratory

Surgical Research Center

Department of Surgery

University of Connecticut

Health Center

Farmington, CT, USA
}

Mansoor Sarfarazi, PhD Surgical Research Center Department of Surgery University of Connecticut Health Center

263 Farmington Ave

Farmington

CT 06030-1110, USA

Tel: +1 (860) 6793629

Fax: +1 (860) 6797524

e-mail:

Mansoor@Neuron.uchc.edu

\begin{abstract}
Molecular genetic studies conducted during the last several years have thrown some light on the basic molecular defects in primary congenital glaucoma (PCG) and the rationale behind the clinical and genetic presentation of this paediatric eye condition. The existence of a hereditary form of PCG segregating as an autosomal recessive trait with high penetrance is now confirmed. The primary molecular defect underlying the majority of PCG cases has been identified as mutations in the cytochrome P4501B1 (CYP1B1) gene. This gene is expressed in tissues of the anterior chamber angle of the eye. Molecular modelling experiments suggest that mutations observed in PCG patients interfere with the integrity of the CYP1B1 molecule as well as its ability to adopt a normal conformation and bind haem. On the basis of these observations, we hypothesised that CYP1B1 participates in the normal development and function of the eye by metabolising essential molecules that are perhaps used in a signalling pathway. Revealing the identity of this molecule is our major objective since it can lead to as yet unknown biochemical cascades controlling the terminal stages of anterior chamber angle development.
\end{abstract}

Key words Congenital glaucoma, Cytochrome p450, Development, Genetics

In 1939, Ringland Anderson ${ }^{1}$ used the following conversation as the prologue to his now classic study Hydrophthalmia or Congenital Glaucoma. The conversation took place in a consulting room in Melbourne, Australia, in 1933, between the father of a 9-year-old boy blind from congenital glaucoma and an ophthalmic surgeon.

Father: Grahame had a trephine operation on each eye when he was a year old. Would we have had a better chance without such treatment?

Surgeon: I do not know.

Father: Do any untreated patients with this disease retain sufficient vision to enable them to earn their living for a few years?

Surgeon: I do not know.
Father: If he marries, will his children be affected?

Surgeon: I do not know.

This conversation outlines in a very simpl way the fears and the questions faced by the patients and their families. It also illustrates the limited help available at the time. The following review is an attempt to evaluate the answers available to us 67 years later.

\section{Definition}

The hallmark of glaucoma is the hourglass pattern of optic nerve atrophy combined with preferential loss of the larger ganglion cells of the retina. ${ }^{2}$ It is currently considered that a variety of aetiological factors, acting individually or in a multifactorial fashion, are capable of triggering pathogenetic cascades leading to these lesions. The severe congenital form of glaucoma is due to obstruction of the drainage of the aqueous humour caused by a primary developmental anomaly at the angle of the anterior chamber. ${ }^{3-8}$ The disease has an onset in the neonatal or infantile period, manifested by symptoms of increased intraocular pressure (IOP) and corneal oedema such as excessive tearing, photophobia and an enlargement of the globe (buphthalmos). The term primary congenital glaucoma (PCG) is reserved for cases where the only anatomical defect observed is isolated trabeculodysgenesis This form of glaucoma is classified as isolated congenital glaucoma according to the Shaffer and Weiss classification, ${ }^{9}$ and as an isolated trabeculodysgenesis according to the anatomical classification of developmental glaucomas presented by Hoskins et al. ${ }^{10}$

\section{Aetiology and pathogenesis}

The tissues forming the anterior segment of the eye are almost exclusively of neural crest origin. ${ }^{11}$ Neural crest cells also contribute to numerous non-ocular structures such as craniofacial and cervical skeleton, ligaments and ganglia of craniofacial nerves, to name but few. In PCG, however, the only observable abnormalities are restricted to the anterior chamber angle, which from an evolutionary 
point of view is also one of the most recently modified regions of the eye. This indicates that the molecular defect(s) underlying PCG interfere with the most terminal and evolutionarily young stages of neural crest cell differentiation. Some of the anterior chamber abnormalities reported in patients with congenital glaucoma include: (a) A 'shagreened membrane' that covers the angle; ${ }^{4}$ (b) failure of separation of the iris and ciliary body from the trabecular fibres, ${ }^{5,8}$ (c) insertion of the longitudinal and circular ciliary muscle fibres into the trabecular fibres anterior to the scleral spur; ${ }^{6}$

(d) abnormally forward insertion of the iris; ${ }^{5,6,8}$ and

(e) thickened trabecular beams. ${ }^{8}$ Naturally, many authors have used these observations as clues to formulation of theories about the aetiology and pathogenesis of congenital glaucoma.

Barkan ${ }^{4}$ believed that the aqueous humour outflow is blocked by impermeable endothelial membrane. Maumenee ${ }^{6}$ deliberated that the abnormal insertion of the ciliary muscle fibres would interfere with the increase in the filtration area produced by the posterior displacement of the scleral spur. Anderson ${ }^{8}$ noted that in congenital glaucoma the trabecular meshwork is not completely uncovered and exposed to the anterior chamber because the iris and anterior ciliary body occupy a position equivalent to the seventh or eighth month of fetal development. He attributed this to premature or excessive formation of unyielding collagenous beams obstructing the posterior sliding of the uveal tissues during the formation of the anterior chamber angle. Earlier authors considered atrophy or cleavage to be the processes underlying the deepening of the anterior chamber angle. ${ }^{5}$

The ultimate cause of the observed anomalies, however, has remained speculative because it was difficult to trace back the proposed pathogenetic mechanisms to some initial, triggering, discrete molecular defect. Such an opportunity came from studies of a completely different aspect of the disease: its genetic nature.

\section{Genetics}

According to Westerlund ${ }^{12}$ the first allusion to an accumulation of familial cases of congenital glaucoma was made by Grelios in 1836 when he noted that the disease occurs endemically in the Jewish population in Algiers. Jungken in 1842 documented a Swedish family in which seven brothers were affected by congenital glaucoma while the parents and two sisters had normal eyes - an obvious case for an autosomal recessive inheritance. These early observations have captured two defining features of PCG as an inherited disorder. First, it is now well established that the incidence of PCG may vary substantially from one population to another. The highest reported incidene of 1:1250 is observed among the Slovakian Roms. ${ }^{13}$ In Europe, incidences from 1:5000 (Switzerland) to 1:22 000 (Slovakia) have been reported, with 1:10 000 being the average number. ${ }^{13-15}$ Second, in most of the familial cases reported to date, this condition is inherited in an autosomal recessive fashion. This is especially true for ethnic groups with high incidence of the disease such as Roms, Turks and Saudi Arabians. ${ }^{13,16,17}$ The disease is familial in $10-40 \%$ of cases, transmission being that of an autosomal recessive with variable penetrance $(40-100 \%) \cdot{ }^{12-15,17}$ Parental consanguinity is also reported for many familial cases. Furthermore, there is a high rate of concordance among monozygotic twins as well as discordance among dizygotic ones. ${ }^{13-15,18}$

The autosomal recessive mode of inheritance has been questioned by some authors. ${ }^{19,20}$ This was mainly based on the observation of unequal sex distribution among affected individuals, with boys being affected nearly twice as frequently as girls, and the lower than expected number of affected sibs in familial cases. In addition, several pedigrees were reported in which the disease was transmitted in successive generations, thus suggesting an autosomal dominant inheritance. ${ }^{18}$ Genetic heterogeneity has been used to explain these observations and discrepancies. ${ }^{13,19-21}$

From the material reviewed so far, one can appreciate that tremendous advances in our understanding of PCG have been provided by the classic and genetic studies. Unfortunately, these two spheres of research remained somewhat isolated from each other. The clinical theories of the disease contributed little to answering the questions raised by the genetic studies, and vice versa. The major reason for this was the lack of knowledge on the primary defect(s) causing this condition. Without clarifying the nature of the initial triggering defect, it is difficult to explain its clinical and genetic presentations and correlate these two into an ordered cause-effect relationship. The introduction in the early 1980s of two new approaches to the study of genetic disorders presented an opportunity to overcome this problem. These were genetic linkage analysis with polymorphic DNA markers and molecular methods for positional cloning of unknown disease genes. ${ }^{22,23}$ In essence, these methods were designed to search for discrete alterations in the genetic material that would be associated with the disease of interest. These methods had two basic requirements: (1) detailed description of the disease phenotype, which would allow clear distinction between normal and affected individuals; and (2) large families, segregating the disease in a Mendelial fashion.

Fortunately, the classic clinical and genetic studies on PCG had previously established and provided these two basic but very necessary elements.

\section{Genetic linkage studies}

In 1995, we applied the methods of genetic linkage analysis to a panel of 17 Turkish PCG families with an autosomal recessive mode of inheritance. Genetic linkage was detected in 11 families between the PCG phenotype and polymorphic DNA markers from the chromosomal region of $2 \mathrm{p} 21 .{ }^{24}$ Haplotype analysis and homozygosity mapping enabled us to map the disease gene within a $2.5 \mathrm{cM}$ interval, flanked by the two DNA markers 
Table 1. CYP1B1 mutations observed in individuals with primary congenital glaucoma

\begin{tabular}{|c|c|c|c|c|}
\hline DNA change & Amino acid change & Location in the molecule & Family origin & References \\
\hline \multicolumn{5}{|l|}{ Missense mutations } \\
\hline TGG $57 \rightarrow$ TGC & Trp 57 $\rightarrow$ Cys & Exon II - hinge region & Hisp-USA & 28 \\
\hline GGA $61 \rightarrow$ GAA & Gly $61 \rightarrow$ Glu & Exon II - hinge region & Turkey, SA & $17,28,31$ \\
\hline CTG $77 \rightarrow$ CCG & Leu $77 \rightarrow$ Pro & Exon II & SA & 31 \\
\hline GGA $281 \rightarrow$ TAA & Glu $281 \rightarrow$ Stop & Exon II & Turkey & 28 \\
\hline GGG $365 \rightarrow$ TGG & Gly $365 \rightarrow$ Trp & Exon III - CCS (J helix) & USA & 28 \\
\hline CGT $368 \rightarrow$ CAT & Arg $368 \rightarrow$ His & Exon III - CCS (J helix) & SA & 31 \\
\hline CCC $379 \rightarrow$ CTC & Pro $379 \rightarrow$ Leu & Exon III - CCS (K helix) & Turkey & 28 \\
\hline GAC $374 \rightarrow$ AAC & Asp $374 \rightarrow$ Asn & Exon III - CCS (K helix) & SA & 17,31 \\
\hline GAA $387 \rightarrow$ AAA & Glu $387 \rightarrow$ Lys & Exon III - CCS (K helix) & Hisp, FrCan, Roms & 28,29 \\
\hline CGC $390 \rightarrow$ CAC & Arg $390 \rightarrow$ His & Exon III - CCS (K helix) & Pakistani & 28 \\
\hline CGC $390 \rightarrow$ AGC & Arg $390 \rightarrow$ Ser & Exon III - CCS (K helix) & SA & 31 \\
\hline CCG $437 \rightarrow$ CTG & Pro $437 \rightarrow$ Leu & Exon III - CCS (meander) & Turkey & 28 \\
\hline CGG $469 \rightarrow$ TGG & Arg $469 \rightarrow$ Trp & Exon III - CCS (haem binding) & Turkey, UK, SA & $17,27,28,31$ \\
\hline \multicolumn{5}{|c|}{ Truncating mutations } \\
\hline Large deletion & & Intron II/Exon III & Turkey & 27 \\
\hline 4238 del 10 & & Exon II & SA & 31 \\
\hline 4306 ins $\mathrm{T}$ & & Exon II & Turkey & 28 \\
\hline 4611 del 9 & SNF 268 del & Exon II & SA & 31 \\
\hline 4668 ins $\mathrm{C}$ & & Exon II & Turkey & 27 \\
\hline 7901 del 13 & & Exon III & Turkey & 27 \\
\hline 8037 dup 10 & & Exon III & US, UK, Turkey & 27,28 \\
\hline 8113 ins $G$ & & Exon III & Japan & 30 \\
\hline $8182 \mathrm{del}$ G & & Exon III & Hisp & 28 \\
\hline 8240 dup 27 & & Exon III & Turkey & 28 \\
\hline
\end{tabular}

DNA alterations are numbered according to the normal sequence of the human CYP1B1 gene (U56438).

CCS, conserved core structures; SA, Saudi Arabian; Hisp, Hispanic; FrCan, French Canadian; Roms, Slovakian Gypsies.

D2S2186 and D2S1346. This PCG locus was designated GLC3A. Inspection of the haplotypes and heterogeneity analysis indicated that six other families were not linked to the GLC3A locus. Subsequent studies found that the disease phenotype in four of these families is linked to other genetic markers spanning a $3 \mathrm{cM}$ interval on chromosome $1 \mathrm{p} 36{ }^{25}$ This genetic interval was defiined by two groups of tightly linked and polymorphic markers: D1S1597/D15485/D1S228 and D1S1176/D1S507/ D1S407. This second locus associated with PCG was designated GLC3B. The remaining two families were not linked to either the GLC3A or GLC3B loci, thus indicating the existence of at least one more PCG locus. The association between the GLC3A locus and PCG phenotype was subsequently confirmed in large panel of families from Saudi Arabia and Slovakian Roms. ${ }^{17,26}$ All families reported in this studies were linked to the GLC3A locus, thus establishing it as a major locus for this condition.

\section{Positional cloning of PCG genes}

The identification of genetic loci associated with the PCG phenotype made it possible to initiate an active search for the actual disease-causing genes and their anticipated defective proteins. In early 1997, we were able to identify the first gene that is directly involved in the pathogenesis of PCG. This gene, the cytochrome P4501B1 (CYP1B1), ${ }^{27}$ has previously been mapped to the $2 \mathrm{p} 21$ region by in situ hybridisation. Our higher-resolution mapping placed this gene within the GLC3A critical candidate region. Sequence analysis of this gene in affected families detected three different DNA mutations. They were not present in the normal population and segregated with disease phenotype in five PCG families previously linked to the GLC3A locus. This study was followed by several other reports on the molecular analysis of PCG families from Turkey, Saudi Arabia, Slovakia (Roms) and Japan. ${ }^{17,28-31}$ Sequence analysis of 116 PCG families revealed extensive allelic heterogeneity in this gene. In 105 families a total of 23 different CYP1B1 mutations were found to segregate with the disease phenotype (Table 1). In 11 families screening of the CYP1B1 coding regions showed no DNA changes. These families may have mutations in promoter or control regions of the gene or may be linked to another congenital glaucoma locus. In all familial cases the mutant alleles cosegregated with the disease phenotype in an autosomal recessive fashion. In three families with only one affected subject, the parents were found to be a normal carriers with the affected child being homozygous or compound heterozygous for the mutant alleles carried by his/her parents. The two sexes were equally represented among the affected individuals. Molecular analysis in cases with parent-child transmission found that in all instances the affected parent was homozygous or compoundheterozygous for the mutant allele(s) while the other parent was a normal gene carrier. Therefore, these are the examples of pseudo-dominant inheritance for an autosomal recessive trait. While in the Turkish and Rom families the observed penetrance was $100 \%$, a reduced penetrance has been reported for the Saudi Arabian families. This was attributed to the possible existence of a dominant modifier locus that is not linked genetically to CYP1B1. Founder effect evidence is presented for the Rom population of Slovakia, where all affected 
individuals identified were homozygous for the Glu387 $\rightarrow$ Lys mutation and also shared an identical haplotype for the six known intragenic single nucleotide polymorphic markers.

In summary, molecular genetic analysis confirmed the existence of a hereditary form of PCG. This condition is inherited as an autosomal recessive trait with high penetrance (73-100\%). The disease is genetically heterogeneous and there are at least three PCGassociated loci in the human genome. However, in the majority of affected subjects (i.e. $87 \%$ of familial and $27 \%$ of sporadic cases) PCG is caused by mutations in the CYP1B1 gene. There are currently not sufficient data to confirm or reject the existence of dominant or multifactorial forms of the disease.

\section{Cytochrome P4501B1 (CYP1B1)}

We thus finally had in our hands the missing piece of the puzzle: a discrete molecular defect capable of initiating pathogenetic responses in the form of a disease we call primary congenital glaucoma. It was hoped that revealing the nature of this triggering event would allow integration of the existing clinical and genetic data, thus producing better understanding of the development and function of the anterior chamber angle. CYP1B1, however, was a quite unexpected gene for congenital glaucoma. Members of the cytochrome P450 family are known mainly for their ability to metabolise xenobiotics. The direct involvement of a cytochrome in human development was rather unexpected. However, careful analysis of the existing data and revival of some old hypotheses regarding the overall role of cytochrome P450s in early development ${ }^{32}$ started to reveal the logic behind this unusual finding. The cytochrome P450 proteins function like any classic enzyme molecules. They carry out a myriad number of oxidative reactions, some of which are biosynthetic, producing necessary hormones or compounds of intermediary metabolism in most living organisms. ${ }^{33}$ Mutations affecting such enzymes generally produce recessive phenotypes because in heterozygous subjects the normal allele is capable of compensating for the mutant one. From this point of view, it is logical that a recessive phenotype such as PCG should be caused by mutation in an enzyme such as CYP1B1.

What exactly is the role of CYP1B1 in normal eye development? CYP1B1 is expressed in the anterior uveal tract of the eye: ciliary body, non-pigmented ciliary epithelium, iris and trabecular meshwork. ${ }^{27,28}$ Recently, we developed a polyclonal anti-CYP1B1-IgG in chicken and were able to demonstrate the presence of CYP1B1 protein in homogenised eye tissues from both rat and mouse (unpublished observation). The uveal tissues have highly specialised functions including accommodation, regulation of outflow facility and formation of aqueous humour, Based on the information available for other members of the P450 family, it is reasonable to expect that CYP1B1 may influence these processes by participating in the metabolism of small biologically active molecule(s) such as steroids and/or arachidonic acid derivatives. These molecules, however, are metabolised by organised cascades of enzymes. Therefore, CYP1B1 may belong to an as yet unknown, endogenous biochemical pathway involved in terminal maturation of the anterior chamber angle.

We have recently presented a molecular model that provides some clues towards the mechanism by which observed mutations in PCG individuals may interfere with normal function of the CYP1B1 molecule. Membrane-bound cytochromes such as CYP1B1 share similar molecule structure. ${ }^{34} \mathrm{~A}$ transmembrane domain is located at the amino terminal end of the molecule. This is followed by a proline-rich 'hinge' region which permits flexibility between the membrane-spanning domain and cytoplasmic portion of the molecule. Induced mutations in the hinge region have previously been reported to interfere with the proper folding and haem-binding properties of other cytochrome P450 molecules. ${ }^{35,36}$ The carboxy-terminal ends are highly conserved among different members of the cytochrome P450 superfamily. They contain a set of conserved core structures (CCS) responsible for the haem-binding ability of these molecules. ${ }^{34}$ Between the hinge and the CCS lies a less conserved substrate-binding region.

In order to evaluate the missense mutations reported in patients with PCG we tried to map them against a three-dimensional model of the CYP1B1 molecule constructed by homology modelling. Our data indicate that the missense mutations affect highly conserved amino acid residues located predominantly either in the hinge region or the CCS part of the CYP1B1 molecule. These mutations are therefore expected to interfere with fundamental properties of the cytochrome P450 molecule such as proper folding, haem binding, substrate accommodation and interaction with the redox partner. Another group of mutations was predicted to introduce premature stop codons by frameshifts in the CYP1B1 open reading frame. These mutations would eliminate at least the haem-binding region of CYP1B1, which is essential for the normal function of every P450 molecule. Therefore, it is expected that these mutations would result in functional null alleles. Alternatively, these mutations may interfere with RNA metabolism by the nonsense-mediated mRNA-decay mechanism.

Preliminary data from site-directed mutagenesis experiments currently being conducted in our laboratory indicate that point mutations in both the hinge region and CCS of CYP1B1 interfere with formation of a stable haemoprotein complex.

\section{Current studies}

As mentioned above, we have hypothesised that CYP1B1 metabolises a molecule of endogenous origin, such as a steroid, fatty acid or prostanoid compound, that is essential for the normal development and function of the eye. The action of CYP1B1 may have two alternative consequences (Fig. 1): it may produce an active compound which, in turn, can act on some unknown 


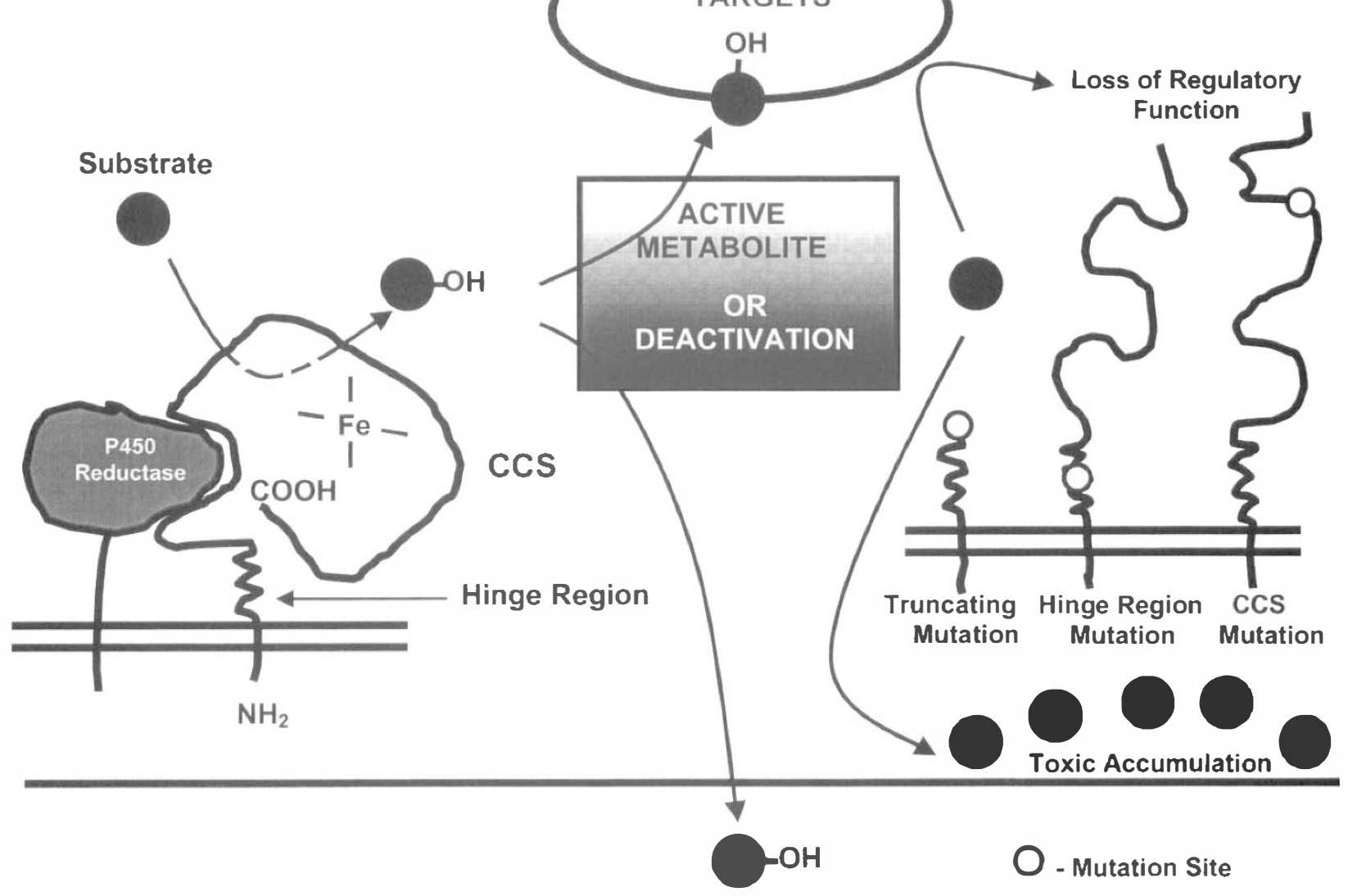

Fig. 1. A cartoon representation of the wild-type and mutant forms of the CYP1B1 molecule and its potential targets. (A) The wild-type CYP1B1 molecule is anchored on the membrane of the endoplasmic reticulum. Its normal functioning requires the assistance of a redox partner, $P 450$ reductase. The P450s have the ability to insert an atom of molecular oxygen into their substrates. This has two possible consequences: (B) In one case the substrate is metabolically activated and acts upon an unknown downstream target. Alternatively, the introduction of molecular oxygen may increase the hydrophilicity of this substrate and facilitates its excretion and clearance from the cell or the site of its normal expression. CYP1B1 mutations may therefore have two alternative consequences. (C) The spatial and temporal regulation of genes controlling the anterior chamber angle development may be altered by the absence of a regulatory molecule (such as steroid) produced by CYP1B1. Alternatively, the signs of developmental arrest observed in the angle of primary congenital glaucoma patients may reflect the toxic effect of a metabolite which is normally eliminated and removed by the CYP1B1 molecule.

downstream targets, or it may deactivate other biologically active substance(s) and assist in discharging them from their active expression site. Revealing the identity of the CYP1B1 substrate is one of the most interesting questions in the field of glaucoma research today since it can direct us to as yet unknown biochemical cascade(s) controlling the terminal stages of anterior chamber angle development. In order to achieve this, first the substrate specificity of CYP1B1 has to be elucidated. One would expect that CYP1B1 catalyses various chemical modifications to a wide range of substrates (steroid, fatty acid or prostanoid compounds). In fact, it has been reported that this enzyme can metabolise $17 \beta$-oestradiol and retinoic acid. ${ }^{37,38}$ We have successully used a heterologous expression system to express large amounts of recombinant CYP1 B1 protein in E. coli. ${ }^{39}$ The recombinant protein is now being utilised in a reconstituted in vitro CYP1B1 monooxygenase system. Using this system, we were able to demonstrate that CYP1B1 is also capable of metabolising progesterone and testosterone (unpublished observation). After the various biochemical activities of CYP1B1 have been fully catalogued, they can be evaluated for the overall efficiency and localisation in space and time during the developmental processes. This will be helpful in identifying specific substrate(s) involved in the development of the eye. Mouse models are useful for determining the CYP1B1 expression profile during various stages of eye development and linking this to the presence of a metabolic intermediate necessary for normal development of the eye. Full phenotypic characterisation of a CYP1B1-null mouse ${ }^{40}$ is currently under way in several laboratories. As mentioned previously, since the anterior chamber angle has undergone some very recent evolutionary changes, this may be a potential problem in using the animal models. For example, only humans and higher apes have the typical trabecular type meshwork while in lower organisms only a reticular meshwork is present. ${ }^{41}$ 


\section{Conclusion}

At the end of his study ${ }^{1}$ Anderson concluded that uncertainty regarding the ultimate cause of congenital glaucoma will persist 'as long as so much concerning the influence of heredity and the forces in prenatal life that make for maldevelopment continues to be wrapped in mystery'. Molecular genetic studies conducted during the last several years have thrown some light on the questions raised by the classic genetic and clinical studies. The existence of hereditary forms of PCG segregating as an autosomal recessive trait with high penetrance has now been confirmed. The primary molecular defect underlying the majority of PCG cases has been determined as mutations in the enzyme cytochrome P4501B1. The CYP1B1 gene has been found and reported to be expressed in the tissues of the anterior chamber angle of the eye. Molecular modelling experiments have suggested that the mutations observed in PCG patients interfere with the integrity of the CYP1B1 molecule as well as its ability to adopt normal conformation and to bind haem. Based on these observations, we hypothesise that CYP1B1 participates in the normal development and function of the eye by metabolising essential molecules that are probably used in a signalling pathway. Revealing the identity of this molecule is our major objective because it can lead us to as yet unknown biochemical cascades controlling the terminal stages of anterior chamber angle development. Deciphering such pathways will improve our understanding of the anterior chamber angle and may lead to the identification of new molecular targets for drug development.

This work was supported by grants (to M.S.) from the National Eye Institute (EY-11095), The Insite Vision, Inc., The Mary Jane \& Peter DaPuzzo Research Fund of The Glaucoma Foundation and the University of Connecticut General Clinical Research Center (M01-RR-06192).

\section{References}

1. Anderson JR. Hydrophthalmia or congenital glaucoma: its causes, treatment and outlook. Cambridge: Cambridge University Press, 1939.

2. Quigley HA. Open-angle glaucoma. N Engl J Med 1993;328:1097-106.

3. De Luise V, Anderson DR. Primary infantile glaucoma (congenital glaucoma). Surv Ophthalmol 1983;28:1-19.

4. Barkan O. Pathogenesis of congenital glaucoma. Am J Ophthalmol 1955;40:1-11.

5. Allen L, Burion HM, Braley AE. A new concept of development of the anterior chamber angle: its relationship to developmental glaucoma and other structural anomalies. Arch Ophthalmol 1955;53:783-98.

6. Maumenee EA. The pathogenesis of congenital glaucoma: a new theory. Trans Am Ophthalmol Soc 1958;56:507-70.

7. Kupfer C, Kaiser-Kupfer M. Observations on the development of the anterior chamber angle with reference to the pathogenesis of congenital glaucomas. Am J Ophthalmol 1979;88:424 6.

8. Anderson DR. The development of the trabecular meshwork and its abnormality in primary infantile glaucoma. Trans Am Ophthalmol Soc 1981;79:458-85.
9. Shaffer RN, Weiss DI. Congenital and pediatric glaucomas. St Louis: Mosby, 1970.

10. Hoskins DH Jr, Shaffer RN, Hetherington J. Anatomical classification of the developmental glaucomas. Arch Ophthalmol 1984;102:1331-6.

11. Johnston MC, Noden DM, Hazelton RD, Coulmbre JL, Coulmbre AJ. Origins of avian ocular and periocular tissues. Exp Eye Res 1979;29:27-43.

12. Westerlund E. Clinical and genetic studies on the primary glaucoma diseases. Copenhagen: NYT Norsdic Forlag, Arnold Busck, 1947.

13. Gencik A. Epidemiology and genetics of primary congenital glaucoma in Slovakia: description of a form of primary congenital glaucoma in gypsies with autosomal recessive inheritance and complete penetrance. Dev Ophthalmol 1989;16:75-115.

14. Francois J. Heredity in ophthalmology. St Louis: Mosby, 1961:218-25.

15. Francois J. Congenital glaucoma and its inheritance. Ophthalmologica 1972;181:61-73.

16. Turacli ME, Aktan SG, Sayli BS, Akarsu AN. Therapeutical and genetical aspects of congenital glaucomas. Int Ophthalmol 1992;16:359-62.

17. Bejjani BA, Lewis RA, Tomey KF, Anderson KL, Dueker DK, Jabac $M$, et al. Mutations in CYP1B1, the gene for cytochrome P4501B1, are the predominant cause of primary congenital glaucoma in Saudi Arabia. Am J Hum Genet 1998;62:325-33.

18. Duke-Elder S. Congenital deformities. In: Duke-Elder S. System of ophthalmology. St Louis: Mosby, 1969:548-65.

19. Jay MR, Rice NSC. Genetic implications of congenital glaucoma. Metab Ophthalmol 1978;2:257-8.

20. Demenias F, Bonaiti C, Briard ML, Feingold J, Frezal J. Congenital glaucoma: genetic models. Hum Genet 1979;46:305-17.

21. Gencik A, Gencikova A, Gerinec A. Genetic heterogeneity of congenital glaucoma. Clin Genet 1980;17:241-8.

22. Botstein D, White RL, Skolnik M, Davis RW. Construction of a genetic linkage map in man using restriction fragment length polymorphisms. Am J Hum Genet 1980;32:314-31.

23. Green ED, Olson MV. Chromosomal region of the cystic fibrosis gene in yeast artificial chromosomes: a model for human genome mapping. Science 1990;250:94-8.

24. Sarfarazi M, Akarsu AN, Hossain A, Turacli ME, Aktan SG Barsoum-Homsy M, et al. Assignment of a locus (GLC3A) for primary congenital glaucoma (buphthalmos) to 2p21 and evidence for genetic heterogeneity. Genomics 1995;30:171-7.

25. Akarsu AN, Turacli ME, Aktan GS, Barsoum-Homsy M, Chevrette L, Sayli SA, et al. A second locus (GLC3B) for primary congenital glaucoma (buphthalmos) maps to the 1 p36 region. Hum Mol Genet 1996;5:1199-203.

26. Plasilova M, Ferakova E, Kadasi L, Polakova H, Gerinec A, Ott $\mathrm{J}$, et al. Linkage of autosomal recessive primary congenital glaucoma to the GLC3A locus in Roms (Gypsies) from Slovakia. Hum Hered 1998;48:30-3.

27. Stoilov I, Akarsu AN, Sarfarazi M. Identification of three different truncating mutations in the cytochrome P4501B1 (CYP1B1) gene as the principal cause of primary congenital glaucoma (buphthalmos) in families linked to the GLC3A locus on chromosome 2p21. Hum Mol Genet 1997;6:641-7.

28. Stoilov I, Akarsu AN, Alozie I, et al. Sequence analysis and homology modelling suggest that primary congenital glaucoma on 2 p21 results from mutations disrupting either the hinge region or the conserved core structures of cytochrome P4501B1. Am J Hum Genet 1998;62:573-84.

29. Plasilova M, Stoilov I, Sarfarazi M, Kadasi L, Ferakova E, Ferak V. Identification of single ancestral CYP1B1 mutation in Slovak gypsies (Roms) affected with primary congenital glaucoma. J Med Genet 1999;36:290-4. 
30. Kakiuchi T, Isashiki Y, Nakao K, Sonoda S, Kimura K, Ohba $\mathrm{N}$. A novel truncating mutation of cytochrome P4501B1 (CYP1B1) gene in primary infantile glaucoma. Am J Ophthalmol 1999;128:370-2.

31. Bejjani BA, Stockton DW, Lewis RA, Tomey KF, Dueker DK, Jabak $\mathrm{M}$, et al. Multiple CYP1B1 mutations and incomplete penetrance in an inbred population segregating primary congenital glaucoma suggest frequent de novo events and a dominant modifier locus. Hum Mol Genet 2000;9:367-74.

32. Nebert DW. Proposed role of drug-metabolizing enzymes: regulation of steady state levels of the ligands that affect growth, homeostasis, differentiation, and neuroendocrine functions. Mol Endocrinol 1991;5:1203-14.

33. Nelson DR, Koymans L, Kamataki T, Stegeman JJ, Feyereisen $\mathrm{R}$, Waxman DJ, et al. P450 superfamily: update on new sequences, gene mapping, accession numbers and nomenclature. Pharmacogenetics 1996;6:1-42.

34. Graham-Lorence SE, Peterson JA. Structural alignments of P450s and extrapolation of the unknown. Methods Enzymol 1996;272:315-26.
35. Yamazaki S, Sato K, Suhara K, Sakaguchi M, Michara K, Omura T. Importance of the proline-rich region following signal-anchor sequence in the formation of correct conformation of microsomal cytochrome P-450s. J Biochem 1993;114:652-7.

36. Chen CD, Kemper B. Different structural requirements at specific proline rich residue positions in the conserved proline-rich region of cytochrome P4502C2. J Biol Chem 1996;271:28607-11.

37. Hayes CL, Spink DC, Spink BC, Cao JQ, Walker NJ, Sutter TR. 17ß-Estradiol hydroxylation catalyzed by cytochrome CYP1B1. Proc Natl Acad Sci USA 1997;93:9776-81.

38. Chen H, Howald WN, Juchau MR. Biosynthesis of all-transretinoic acid from all-trans-retinol: catalysis of all-transretinol oxidation by human p-450 cytochromes. Drug Metab Disp 2000;28:315-22.

39. Jansson I, Stoilov I, Sarfarazi M, Schenkmann JB. Enhanced expression of CYP1B1 in E. coli. Toxicology 2000;144:211-9.

40. Buters JT, Sakai S, Richter T, Pineau T, Alexander DL, Savas $\mathrm{U}$, et al. Cytochrome-450 CYP1B1 determines susceptibility to 7,12-dimethylbenz [a]anthracene-induced lymphomas. Proc Natl Acad Sci USA 1999;96:1977-82.

41. Rohen JW. The histologic structure of the chamber angle in primates. Am J Ophthalmol 1961;52:529. 